\title{
Experimental investigation of precoding for EM exposure reduction
}

\author{
P. Chambers, T.W.C. Brown \\ Centre for communications systems research (CCSR), University of Surrey, \\ Guildford, Surrey, UK. \{P.Chambers, T.Brown\}@surrey.ac.uk
}

\begin{abstract}
Reduction of human exposure to electromagnetic (EM) radiation from communications devices without compromising link quality is of importance as people spend more and more time using devices with wireless connectivity. This paper considers the idea of designing a low complexity baseband precoder to reduce user EM exposure for the user scenario of a laptop making an uplink connection to an access point terminal in a picocell environment. The design of the precoder is supported by channel sounding measurements and a ten-fold reduction in user exposure is indicated.
\end{abstract}

Index Terms-Exposure Index, SAR, MIMO, Channel Measurements, Beamforming, Precoding.

\section{INTRODUCTION}

Although as communications standards progress, there has been a trend in maintaining a reasonably consistent transmit power level with both $3 \mathrm{G}$ and $4 \mathrm{G}$ systems using $23 \mathrm{dBm}$ [1], the increased use of portable devices with wireless connectivity is considerable. Laptops and now the newer tablet devices all signal increased user EM exposure due to the simple reason that, because of the sheer popularity of such devices, people are spending more time in proximity to wireless transmissions. In order to limit exposure to EM transmissions, an ideal solution would be to somehow reduce the emission power while maintaining the same signal-to-noise ratio (SNR) at the receiver. This has the effect of reducing the specific absorption ratio (SAR), which is metric that is a measure of user exposure. Some current approaches in [2] [3], consider a hardware based approach where antennas are designed in conjunction with phase splitters to steer a null towards the user. In [4], a signal processing approach, referred to as SAR codes, is proposed based on the idea that a portable device placed in a 'talk position', i.e. placed at the user's skull, can reduce its userimparted power by setting a phase offset of $\frac{\pi}{3}$ between the transmit spatial samples. This phase offset was calculated based on CST microwave studio software simulations, which incorporate dual antennas in conjunction with a human skull phantom.

This work considers the user scenario of the user being situated at a laptop that is making transmissions to an access point terminal in an indoor picocell environment. In contrast to the CST software approach of [4], an experimental approach is considered here and unlike the hardware solution described in [2] [3], a low complexity baseband precoding technique is proposed.

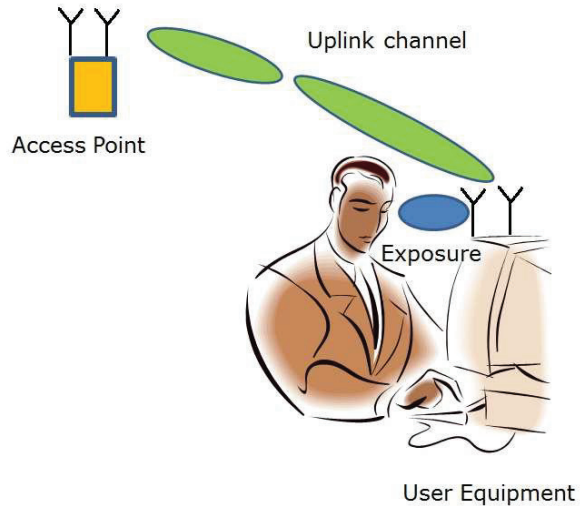

Fig. 1. Illustrative schematic of user scenario depicting user exposure during uplink.

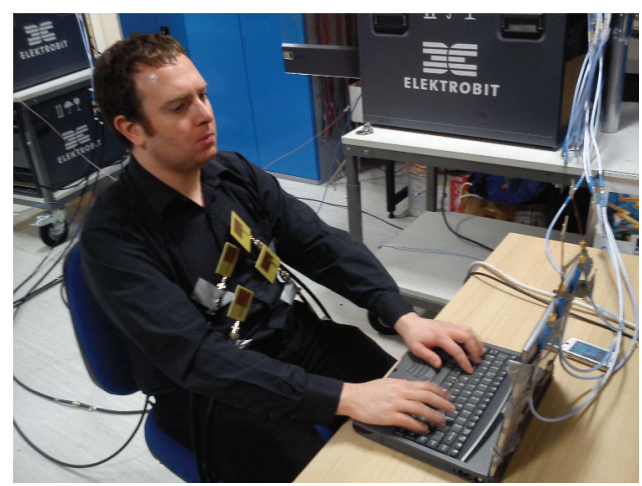

Fig. 2. Measurement campaign: Laptop making uplink with access point and user with probe.

\section{USER SCENARIO}

\section{A. Experimental approach}

The user scenario upon which the experimental work is based is depicted illustratively in Fig. 1. There is an uplink communications channel between a user equipment and an access point while simultaneously there is EM exposure to the user.

In order to investigate this user scenario, a measurement campaign is developed and is depicted in Fig. 2. Here, a laptop (Tx) is equipped with two pairs of two antennas, each making the uplink transmission to an access point $(\mathrm{Rx})$, which is also equipped with two antennas. Two pairs of transmit antennas, spaced at approximately $\frac{\lambda}{2}$ with respect to a carrier frequency, 
TABLE I

MEASUREMENT PARAMETERS

\begin{tabular}{||l||l||}
\hline Parameter & Value \\
\hline Tx Power & $-10 \mathrm{dBm}$. \\
Bandwidth & $10 \mathrm{MHz}$. \\
MIMO sampling frequency & $24.495395 \mathrm{~Hz}$. \\
Carrier frequency, $f_{c}$ & $2.3 \mathrm{GHz}$. \\
Measurement time & $\approx 7 \mathrm{Mins}$. \\
Antenna spacing & $\approx \frac{\lambda}{2}$ (two pairs). \\
\hline
\end{tabular}

$f_{c}$, of $2.3 \mathrm{GHz}$, are used in order to demonstrate repeatability and the user is considered to be in the far field region. It should be noted that unlike the approach in [5] this user scenario does not consider the idea of a user having a laptop on their person, i.e. on their lap, in which case arguably the user could be in the near field. Also, although an uplink receiver at the access point is integral to the user scenario, the focus of the analysis in this work is the channel between either pair of the two antenna laptop and a probe channel. The probe channel is an array of four receive antennas strategically placed on the user's chest (two on upper chest, two on lower chest) in order to provide a measure of how much EM energy would be transmitted to the user, i.e. a measure of user EM exposure. It should be noted that throughout the measurement campaign, the user is seated in front of the laptop, as shown in the photo, and is free to move over a small distance, as would normally occur as a result of human posture changes. This will of course give rise to a certain degree of fading.

\section{B. Measurement parameters and post-processing}

Channel measurements are recorded over approximately a seven minute period and the system parameters for the user scenario measurement campaign are given in Table I.

The channel measurements are made using an Elektrobit Propsound wideband MIMO channel sounder, which uses a direct-sequence spread spectrum signal produced from binary phase shift keyed modulated pseudo-noise codes. These codes ascertain the channel impulse response by using a correlation based method and as a result, the frequency domain response of the measurements assumes a sinc function response of the form [6]: $\operatorname{sinc}\left(f / f_{\mathrm{Clk}}\right)$, where $f$ is the frequency domain increment over the measurement bandwidth and $f_{\mathrm{Clk}}=16 \mathrm{MHz}$ refers to the clock frequency of the channel sounder circuitry. The experimental investigation in this work requires an accurate channel impulse response and thus all measurements are transformed into the frequency domain where an appropriate inverse sinc function is applied before transforming back to the time domain.

\section{SySTEM MODEL}

In order to develop the case for a baseband precoder that reduces user EM exposure, consider firstly the complex envelope of a series of upconverted received signal samples, $y(t)$, where $t$ is the sample time, defined as [7]:

$$
y(t)=\Re\left\{x(t) \otimes h(t) \exp \left(j 2 \pi f_{c} t\right)\right\} .
$$

The notation $\Re\{\cdot\}$ refers to the real part of a complex number, $\otimes$ is the convolution operator and $j=\sqrt{-1} . x(t)$ is the time domain baseband transmit sample, $h(t)$ is the time domain baseband channel impulse response and $f_{c}$ is the carrier frequency. Further to this, a phase shifted version of $y(t)$, i.e., $y(t)$, may also be defined:

$$
\tilde{y(t)}=\Re\left\{x(t) \exp (j \theta) \otimes h(t) \exp \left(j 2 \pi f_{c} t\right)\right\},
$$

where $\theta$ is the associated phase shift. Clearly, a phase shifted version of the upconverted receive signal, $y(t)$, can be achieved by simply applying a factor $\exp (j \theta)$ to the associated baseband signal $x(t)$. Given a scenario where there are $N_{T}$ transmit RF chains, each being fed an equivalent baseband sample, $x(t)$ and employing frame based transmission, with $N_{S}$ samples per frame, onto a block-fading channel then the transmit signal in time and space would be a matrix, $\mathbf{X}$, of dimension: $N_{S} \times N_{T}$. From a practical point-of-view, in order to apply relative phase shifts in the manner implied by eqns (1) and (2), a matrix, $\tilde{\mathbf{X}}$ is now defined:

$$
\tilde{\mathbf{X}}=\mathbf{X P},
$$

where $\mathbf{X}$ is also a $N_{S} \times N_{T}$ matrix with each of $N_{T}$ columns containing samples implied by the product: $x(t) \exp \left(j 2 \pi f_{c} t\right)$, i.e., are upconverted transmissions of baseband samples: $x(t)$, and $\mathbf{P}$ is defined:

$$
\left[\begin{array}{ccccc}
1 & 0 & \cdots & \cdots & 0 \\
0 & \exp \left\{j \theta_{2}\right\} & & & \vdots \\
\vdots & & \exp \left\{j \theta_{3}\right\} & & \vdots \\
\vdots & & & \ddots & 0 \\
0 & \cdots & \cdots & 0 & \exp \left\{j \theta_{N_{T}}\right\}
\end{array}\right] .
$$

$\mathbf{P}$ is a unitary matrix, i.e. $\mathbf{P} \mathbf{P}^{H}=\mathbf{I}$ where $(\cdot)^{H}$ denotes the matrix Hermitian transpose and $\mathbf{I}$ is the identity matrix. $\mathbf{P}$ is of dimension: $N_{T} \times N_{T}$ and its design is the subject of Section IV. If there are $N_{\text {Probe }}$ probe antennas present on the user, then an average measure of power over the probe antennas as function of angles, i.e., $r\left(\theta_{2}, \theta_{3}, \ldots, \theta_{N_{T}}\right)$, as a result of applying the precoder, $\mathbf{P}$, may be written as:

$$
\begin{aligned}
r\left(\theta_{2}, \theta_{3}, \ldots, \theta_{N_{T}}\right)= & \frac{1}{N_{\text {Probe }}} \sum_{i=1}^{N_{\text {Probe }}} \operatorname{Var}_{t}\left[\sum_{k=1, \theta_{1}=0}^{N_{T}} \Re\{x(t)\right. \\
& \left.\left.\exp \left(j \theta_{k}\right) \otimes h_{i, k}(t) \exp \left(j 2 \pi f_{c} t\right)\right\}\right] .
\end{aligned}
$$

The notation, $\operatorname{Var}_{t}[\cdot]$, refers to the statistical variance of the collection of convolved receive samples, each defined at the sample time, $t$, that arise when the transmit data frame is convolved with the channel impulse response. $h_{i, k}$ denotes the channel impulse response between the $k^{\text {th }}$ transmitter and $i^{t h}$ probe antenna. 
TABLE II

TRANSMIT SIGNAL PARAMTERS.

\begin{tabular}{||l||l||}
\hline Parameter & Value \\
\hline Tx Power & $23 \mathrm{dBm}$. \\
Signal bandwidth & $5 \mathrm{MHz}$. \\
Signal sampling frequency & $7.68 \mathrm{MHz}$. \\
Signal frame length & $10 \mathrm{mSec}$. \\
Fast Fourier transform (FFT) size & 512. \\
Number of samples per frame, $N_{S}$, & 7680. \\
\hline
\end{tabular}

It should be understood at this point that the samples in the columns of the transmit matrix, $\mathbf{X}$, are identical and thus it is implicit that this form of precoding is suited to be used in conjunction with beamforming. In the single-user MIMO case, the optimal transmit strategy from a capacity point of view is spatial multiplexing by using precoders based on the singular value decomposition (SVD) of MIMO channel matrix and then performing spatial waterfilling. However, it has been shown in [8] [9] that, in the multiuser scenario, the use of beamforming does in fact lead to high data throughputs in the uplink. It was then proven mathematically rigorously in [10] that beamforming is in fact optimal in a capacity sense for the case of the uplink of multiuser systems. On the basis of this, the precoder structure proposed here, i.e. P, could be fedback to the user terminal (transmitter) by exploiting pilot based channel knowledge at the access point (receiver). Furthermore at the access point itself, $\mathbf{P}$ could then be matched with $\mathbf{P}^{H}$ thus allowing multiuser beamforming precoders to be used without performance degradation. To clarify, while the design of $\mathbf{P}$ appears fixed in this work, in reality in a practical implementation, $\mathbf{P}$ would have to interact with the (multiuser) beamforming precoder, which is changing on each channel estimation. As a result, so too would $\mathbf{P}$ have to change in accordance in order to meet an effective angular design criterion, which will be described in Section IV.

\section{ANALYSIS AND PRECODER DESIGN}

As was indicated in Section II, the specific case where $N_{T}=2$ and $N_{\text {Probe }}=4$ is considered in the context of two separate transmit antenna pairs for repeatability. For the 2 transmit RF chain sample streams, the baseband samples, indicated by the matrix $\mathbf{X}$, were orthogonal division multiplexing (OFDM) symbols derived from the link level long term evolution (LTE) simulator in [11]. Table II summarises the transmit signal parameters [12].

Notably in Table II, there is an increase in transmit signal power to $23 \mathrm{dBm}$ by comparison to the lower value of $-10 \mathrm{dBm}$ in the case of the channel measurements in Section II. This was done in order to effectively simulate $3 \mathrm{G}$ and $4 \mathrm{G}$ standards' transmit power as mentioned in [1] and this power level was split equally over the two transmit RF chains. For either transmit antenna pair, the pair of OFDM frame copies in $\mathbf{X}$ are upconverted and convolved with channel impulse response measurements described in Section II and denoted $h_{i, k}$ in Section III. Throughout this analysis, appropriate subscript notation, i.e. $(\cdot)_{12}$ and $(\cdot)_{34}$, will be used where necessary

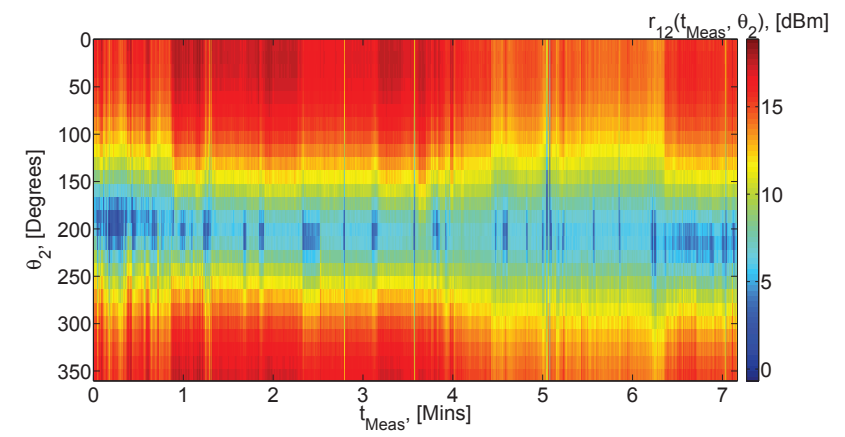

Fig. 3. Plot of $r_{12}\left(t_{\text {Meas }}, \theta_{2}\right)$ in $\mathrm{dBm}$ indicating appropriate design of $\mathbf{P}$.

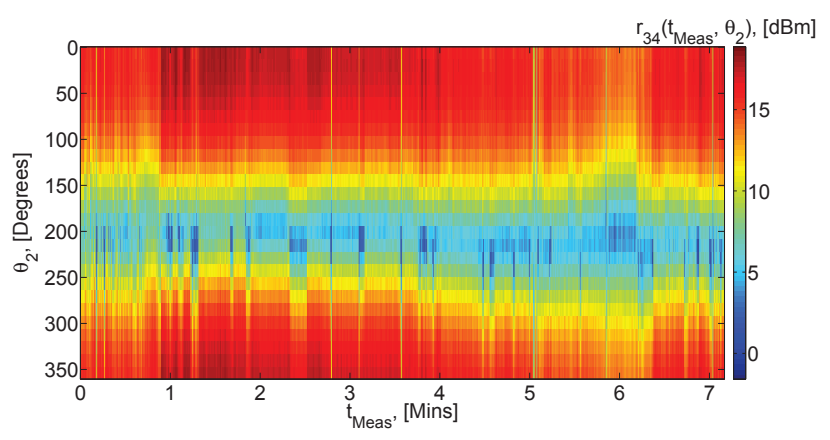

Fig. 4. Plot of $r_{34}\left(t_{\mathrm{Meas}}, \theta_{2}\right)$ in $\mathrm{dBm}$ indicating appropriate design of $\mathbf{P}$.

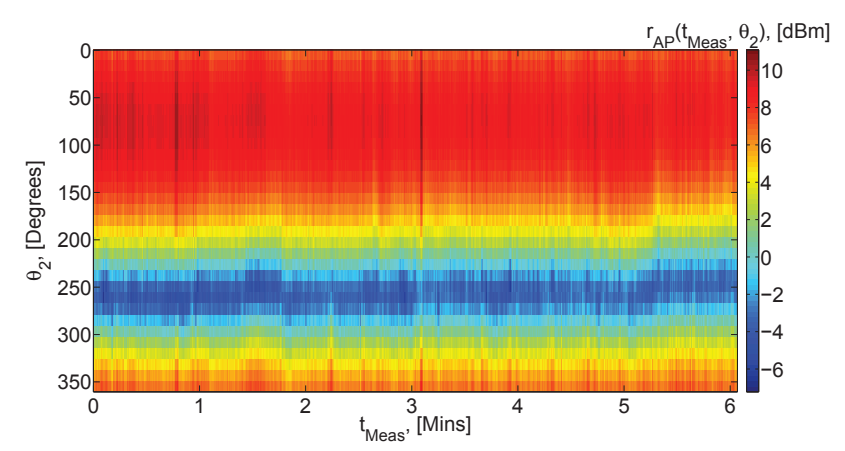

Fig. 5. Plot of $r_{A P}\left(t_{\text {Meas }}, \theta_{2}\right)$ in $\mathrm{dBm}$ indicating the effect of $\mathbf{P}$ to the uplink channel.

in order to distinguish between transmit antenna either pair and there will be also be a comparison to the case of applying the same precoding analysis to channels in the uplink from the user terminal to the access point denoted by $(\cdot)_{A P}$. Since by definition, $\theta_{1}=0$, only $\theta_{2}$ is required in this case and thus plots of $r\left(t_{\text {Meas }}, \theta_{2}\right)$ are given for the two transmit antenna pairs in Figs. 3 and 4 . The term, $t_{\text {Meas }}$, is the measurement time increment that is based on the inverse of the MIMO sampling frequency and is distinct from $t$.

Looking at Figs.(3) and (4), the uppermost horizontal segment of the plot corresponding to $\theta_{2}=0$ provides a measure of received power when the precoder is not applied and therefore the comparative reduction in user-imparted power is significant around the $150^{\circ}-200^{\circ}$ region in both cases. The similarity of Figs.(3) and (4) confirms repeatability, while the notable 


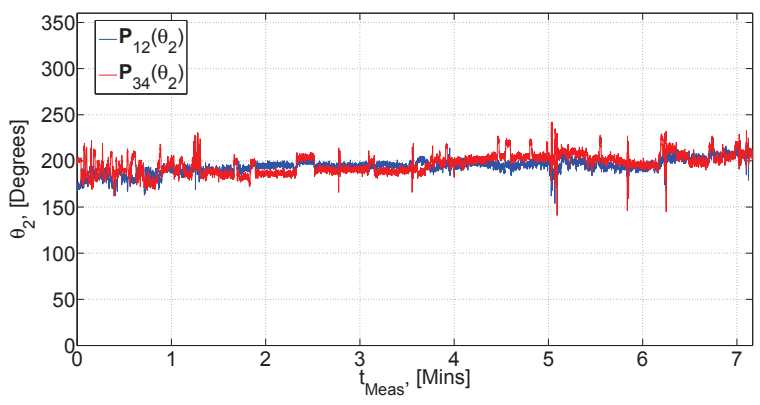

Fig. 6. Plot of $\mathbf{P}_{12}\left(\theta_{2}\right)$ and $\mathbf{P}_{34}\left(\theta_{2}\right)$.

TABLE III

Mean power With and Without implementation of $\mathbf{P}$.

\begin{tabular}{||l||l||}
\hline Precoding & Mean power, $[\mathrm{mWatts}]$ \\
\hline $\mathbf{P}_{12}=\mathbf{I}$ (No precoding) & 39.9 \\
$\mathbf{P}_{12}\left(\theta_{2}\right)$ (Precoding) & 3.99 \\
$\mathbf{P}_{34}=\mathbf{I}$ (No precoding) & 43.1 \\
$\mathbf{P}_{34}\left(\theta_{2}\right)$ (Precoding) & 3.6 \\
\hline
\end{tabular}

contrast of these figures to Fig. (5) offers the reassurance that $\mathbf{P}$, if chosen in the same range of $\left(150^{\circ}-200^{\circ}\right)$, should not compromise the uplink to the access point to badly since the drop in power is not in the same region. Given these observations, it is now necessary to consider a choice of $\theta_{2}$ in order to design $\mathbf{P}$ appropriately, which will be denoted $\mathbf{P}\left(\theta_{2}\right)$ and defined:

$$
\mathbf{P}\left(\theta_{2}\right)=\underset{\theta_{2}}{\arg \min } r\left(t_{\text {Meas, }}, \theta_{2}\right) .
$$

Therefore, a search is performed over the data set in Figs. (3) and (4) to find $\mathbf{P}\left(\theta_{2}\right)$ with the result of this search presented in Fig. (6).

Since $\mathbf{P}_{12}\left(\theta_{2}\right)$ and $\mathbf{P}_{34}\left(\theta_{2}\right)$ are quite similar sets, they can be amalgamated into a common set and choice of $\theta_{2}$ determined as:

$$
\theta_{2}=\operatorname{Mean}\left[\left\{\mathbf{P}_{12}\left(\theta_{2}\right), \mathbf{P}_{34}\left(\theta_{2}\right)\right\}\right],
$$

where Mean $[\cdot]$ denotes the statistical mean and $\{\cdot, \cdot\}$ denotes the union of sets. On applying eqn.(7), $\theta_{2}$ is calculated to be: $\theta_{2}=196^{\circ}$ as a rounded integer.

\section{EXPOSURE REDUCTION}

In order to determine the effectiveness of this choice of $\theta_{2}$ and hence the implementation of $\mathbf{P}$, the cumulative receive power over time, $t_{\text {Meas }}$, for both antenna pairs with and without implementation of $\mathbf{P}$ is plotted in Fig. (7) and the mean power over time, $t_{\text {Meas }}$, again, with and without implementation of $\mathbf{P}$ is given in Table. III. It should be noted that in the results that follow, $\mathbf{P}\left(\theta_{2}\right)$ refers to the implementation of the precoder $\mathbf{P}$ with appropriate choice of angle $\theta_{2}$ while setting $\mathbf{P}=\mathbf{I}$ refers to the conventional case without the precoder. Both Fig. (7) and Table. III indicate a ten-fold reduction in user exposure.

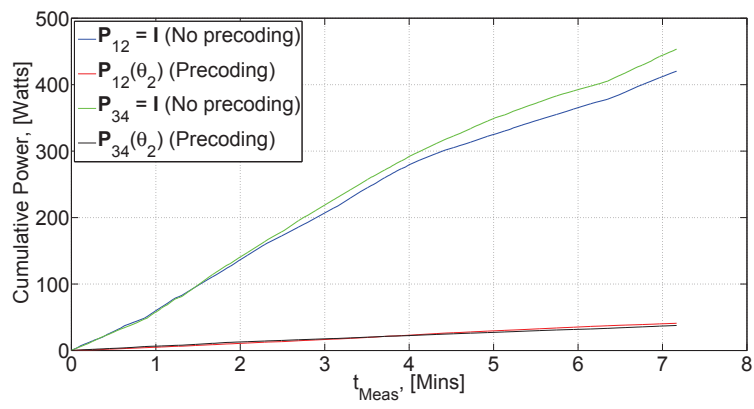

Fig. 7. Cumulative power.

\section{Vi. Conclusions}

In this work, a novel low complexity baseband precoder is proposed that is designed based on an experimental approach that was pertinent to a user scenario where a laptop user makes an uplink to an access point in an indoor picocell environment. A general framework for the design of $\mathbf{P}$ is given and results based on the case where $N_{T}=2$ were presented. The results indicate a ten-fold reduction in user exposure provided of course that the user and the access point would not be colocated. The application of this precoder requires that identical transmit samples appear on all transmit RF chains. As a result, this procoder is suitable to be used in conjunction with signal processing frameworks where (multiuser) uplink beamforming is applied.

\section{ACKNOWLEDGMENTS}

This paper reports work undertaken in the context of the project LEXNET. LEXNET is a project supported by the European Commission in the 7th Framework Programme (GA $\left.n^{\circ} 318273\right)$. For further information, please visit www.lexnetproject.eu

\section{REFERENCES}

[1] B. M. Hochwald and D. J. Love, "Minimizing exposure to electromagnetic radiation in portable devices," in Proc. Information theory and applications workshop (ITA), Feb 2012, pp. 255-261.

[2] K. C. Chim, K. C. L. Chan, and R. D. Murch, "Investigating the impact of smart antennas on SAR," IEEE Trans. Antennas Propag., vol. 52 no. 5, May 2004.

[3] K. C. Chim and R. D. Murch, "Investigating the effect of smart antenna on SAR," in Proc. IEEE Antennas and Propagation Society International Symposium, vol. 1, 2002, pp. 432-435.

[4] B. M. Hochwald, D. J. Love, S. Yan, and J. Jin, "SAR codes," in Proc. Information theory and applications workshop (ITA), Feb 2013, pp. 1-9.

[5] J. Guterman, A. A. Moreira, C. Peixeiro, and Y. Rahmat-Samii, "User interaction with antenna arrays in mimo-enabled laptops," in Proc. 3rd European Conference on Antennas and Propagation(EuCAP 2009), Mar 2009, pp. 226-230.

[6] R. J. Pirkl and G. D. Durgin, "Optimal sliding correlator channel sounder design," IEEE Trans. Wireless Communs., vol. 7, no. 9, Sep 2008.

[7] W. H. Tranter, K. S. Shanmugan, T. S. Rappaport, and K. L. Kosbar, Principles of communication systems simulation with wireless applications. Prentice Hall communications engineering and emerging technologies series, 2004.

[8] W. Rhee and J. M. Cioffi, "On the asymptotic optimality of beamforming in multi-antenna Gaussian multiple access channels," in Proc. IEEE Globecomm Conf., Nov 2001, pp. 891-895. 
[9] W. Yu, W. Rhee, S. Boyd, and J. M. Cioffi, "Iterative water-filling for Gaussian vector multiple access channels," in Proc. Int. Symp. Information Theory (ISIT) 2009, 2001, p. 322.

[10] W. Rhee, W. Yu, and J. M. Cioffi, "The optimality of beamforming in uplink multiuser wireless systems," IEEE Trans. on Communs., vol. 3, no. 1, Jan 2004.

[11] J. Blumenstein, J. C. Ikuno, J. Prokopec, and M. Rupp, "Simulating the long term evolution uplink physical layer," in Proc. 53rd International Symposium ELMAR, Sep 2011, pp. 141-144.

[12] S. Sesia, I. Toufik, and M. Baker, LTE-The UMTS Long Term Evolution: From Theory to Practice. Wiley, 2009. 